\title{
Nonlocal Nonlinear Problems for One-Diménsional Parabolic System
}

E. M. Chrzānowski

Es werden zwei nichtlokale nichtlineare Probleme für ein parabolisches Gleichungssystem betrachtet, und zwar geht es darum, eine Lösung des Systems

$$
\vec{u}_{t}(x, t)=D \vec{u}_{x x}(x, t)+\vec{f}(x, t, \vec{u}(x ; t))
$$

zu finden, die entweder die Bedingungen

$$
\begin{aligned}
& \vec{u}(0, t)=\vec{\varphi}(t) \text { für } \quad t \in\langle\overline{0}, T), \\
& \vec{u}(x, 0)=\vec{\psi}(x) \quad \text { für } \quad x \in\langle 0,1\rangle, \\
& \overrightarrow{\vec{u}}(1, t)-\vec{u}\left(x_{0}, t\right)=\vec{h}\left(x_{0}, t, \vec{u}\left(x_{0}, t\right)\right)
\end{aligned}
$$

oder die Bedingung

$$
\int_{0}^{1} \vec{u}(x, \iota) d x=\ddot{g}(\ell)
$$

erfüllt. Dazu konstruiert man einen Operator $L: C(\bar{\Omega}) \rightarrow C(\bar{\Omega})$ als eine Summe von vier Potentialen und zeigt, daß dieser Operator genau einen Fixpunkt besitzt, der auch einzige Lösung des Problems ist.

Рассматриваются две нелокальные, нелинейне и́роблемы, а именно требуется найдти решение системы параболических уравнений

$$
\vec{u}_{t}(x, t)=D \vec{u}_{x x}(x, t)+\vec{f}(x, t, \vec{u}(x, t)),
$$

удовлетворяющее либо условиям

$$
\begin{aligned}
& \vec{u}(0, t)=\vec{\varphi}(t) . \text { для } \quad t \in\langle 0, T), \\
& \vec{u}(x, 0)=\vec{\psi}(x) \quad \text { для } \quad x \in\langle 0,1\rangle, \\
& \vec{u}(1, t)-\vec{u}\left(x_{0}, t\right)=\vec{h}\left(x_{0}, t, \vec{u}\left(x_{0}, t\right)\right)
\end{aligned}
$$

либо условию

$$
\int_{0}^{1} \vec{u}(x, t)^{\prime} d x=\vec{g}(t) .
$$

Для әтого строится оператор $L: C(\bar{\Omega}) 1 \rightarrow C(\bar{\Omega})$, складываюииися из четырех потенциалов, и доказывается, 'то он имеет единственную неподвижную точку, которая есть также единственнос решение поставлениой проблемы.

In the paper two nonlocal, nonlinear problems for a system of parabolic equations are con. sidered:.

to find ab solution of the system

$$
\vec{u}_{t}(x, t)=D \vec{u}_{x x}(x, t)+\vec{f}(x, t, \vec{u}(x, t))
$$


subject to the conditions

$$
\begin{aligned}
& \vec{u}(0, t)=\vec{\phi}(t), \quad t \in\langle 0, T), \\
& \vec{u}(x, 0)=\vec{\psi}(x), \quad x \in\langle 0,1\rangle, \\
& \vec{u}(1, t)-\vec{u}\left(x_{0}, t\right)=\vec{h}\left(x_{0}, t, \vec{u}\left(x_{0}, t\right)\right)
\end{aligned}
$$

or

$$
\int_{0}^{1} \vec{u}(x, t) d x=\vec{g}(t) .
$$

For this an operator $L: C(\bar{\Omega}) \rightarrow C(\bar{\Omega})$ being a sum of four potentials is constructed. It is shown that the operator $L$ has only one fixed point. Moreover it is proved that the fixed point is the only solution of the considered problem.

\section{Formulation of Prohlem I}

We shall consider the following Problem I: to find solutions of the system

for

$$
\vec{u}_{t}(x, t)=D \vec{u}_{x x}(x, t)+\vec{f}(x, t, \vec{u}(x, t))
$$

$$
{ }^{\prime}(x, \imath) \in \Omega \stackrel{\prime}{=}\{(x, t): x \in(0,1), \iota \in(0, T), T<\infty\} .
$$

subject to the conditions

$$
\begin{aligned}
& \vec{u}(0, t)=\vec{\phi}(t), \quad t \in\langle 0 ; T\rangle, \\
& \vec{u}(x, 0)=\vec{\psi}(x), \quad x \in\langle 0,1\rangle, \\
& \vec{u}(1, t)-\vec{u}\left(x_{0}, t\right)=\vec{h}\left(x_{0} ; t, \vec{u}\left(x_{0}, t\right)\right),
\end{aligned}
$$

where $x_{0}$ is an arbitrary fixed point of the interval $(0,1), D$ is an $n_{\text {-dimensional }}$ scalar matrix, $\vec{\phi}, \vec{\psi}, \vec{h}, \vec{f}$ are $n$-dimensional vector-functions. The boundary condition (1.4) is nonlocal.

The theory of nonlocal linear boundary value problems for single equations has - been developed mainly in the last twenty years by A. V. Bizadse and A. A. Samarski [1], J. A. Roitrerg and 7. G. Sheftel [10], A. F. Nerso [8], A. A. Kerefov [6] and others. A linear problem of type (1.1)-(1.4) has been formulated and solved . recently by M. MAJcinowski [7].

As a physical example of such a situation one can mention the problem of determination conceritrations of $n$-components in the $(n+1)$-component fluid. Concentrations of $n$-components are to be determined from the boundary date (1.2), (1.3) and the nonlocal nonlinear condition (1.4).

\section{Solution of Pròblem I}

By a solution of Problem I we shall mean a vector function $\vec{u}$, continuous in $\bar{\Omega}$, and having continuous derivatives $\vec{u}_{t}, \vec{u}_{x x}$ in $\Omega$, satisfying conditions (1.1)-(1.4).

Problem I will be solved under the following assumptions:

! (a $a_{1}$ System (1.1) is a parabolic system in Petrovski's sense, i.e. for any'eigenvalue $\lambda$ of the matrix $D$ the inequality re $\lambda>0$ is satisfied.

$\left(a_{2}\right) \vec{\varphi}(t)$ is continuous and bounded for $t \in(0, T)$.

、 ( $\left.a_{3}\right) \vec{\phi}(t)$ is piecewise of the class $C^{\prime}(0, T)$. 
$\left(a_{4}\right) \quad \vec{\varphi}^{\prime}(t)$ is bounded for $t \in\left(0, T^{\prime}\right)$.

(a) $\vec{\psi}(x)$ has a bounded variation for $x \in(0,1)$.

$\left(a_{6}\right) \quad \vec{\psi}(x)$ is the sum of its trigonometric Fourier series.

$\left(a_{7}\right)$ For every $x \in(0,1)$, sup $\int_{0}^{1}\left\|\dot{f}_{x x}(x, t, z)\right\| d x<\tilde{C}$ for every $z \in \mathbf{R}^{n}$, where $\tilde{C}$ is a positive constant independently of $z$.

(a $\left.\mathbf{a}_{8}\right) \vec{f}(x, t, z)$ has second derivative with respect to $x$, piecewise continuous for $t$ and $z$ fixed.

(a) $\vec{f}(x, \ell, z)$ is continuous and bounded in

$$
\tilde{\Omega}=\left\{(\dot{x}, t, z): x \in\langle 0,1\rangle, t \in\left(0,{ }^{\prime} T\right), z \in \mathbf{R}^{n}\right\} .
$$

(a $\left.a_{10}\right)\|\vec{f}(x, t, z)-\vec{f}(x, t, \tilde{z})\|_{\mathbf{E}_{n}} \leqq K\|z-\tilde{z}\|_{\mathbf{E}_{n}}$; where $K$ is a positive constant.

$\left(a_{11}\right) \vec{f}(0, t, z)=0$ for every $t \in(0, T)$ and every $z \in \mathbf{R}^{n}$.

$\left(a_{12}\right) \vec{h}\left(x_{0}^{\prime}, t, y\right)$ has for $t \in(0, T)$ and $y \in C^{\prime}$ piccewise continuous first derivatives.

$\left(\mathrm{a}_{13}\right)\left\|\vec{h}\left(x_{0}, t, y\right)-\vec{h}\left(x_{0}, t, y^{\prime}\right)\right\|_{\mathbf{E}_{n}} \leqq H\left\|y-y^{\prime}\right\|_{\mathbf{E}_{n}}$, where $H$ is a positive constant.

$\left(\mathrm{a}_{14}\right) \vec{\psi}(1)-\vec{\psi}\left(x_{0}\right)=\vec{h}\left(x_{0}, 0, \vec{\psi}\left(x_{0}\right)\right)$.

To solve this problem we recall some properties of the fundamental matrix function $\dot{M}$ introduced in [7]. The function $M$ is of the form

$$
M(x, t)=1+2 \sum_{k=1}^{\infty} \exp \left[-k^{2} x^{2} D t\right] \cos k \pi x
$$

and belongs to the class $C^{\infty}$ in the:domain

$$
P=\{(x, t): x \in(-\infty,+\infty), t \in(0,+\infty)\} .
$$

The corresponding partial derivatives can be obtained by the term-by-term differentiation of the series $(1.5)$ and

$$
\lim _{t \rightarrow 0} \frac{\partial^{8} M(x, t)}{\partial x^{s}}=0, \text { for } s=0,1,2, \ldots, x \neq 0,+2,+4,+\cdots
$$

Each column of the matrix function $M$ is a solution of the homogeneous system (1.1).

Theorem 1: If $\vec{u}$ is a solution of Problem $\mathrm{I}$, then $\vec{u}$ is a solution of the system of Volterra integral equations

$$
\begin{aligned}
\vec{u}(x, t)= & \frac{1}{2} \int_{0}^{t} \int_{0}^{i}[M(x-s, t-\eta)-M(x+s, t-\eta)] \vec{f}(s, \eta \vec{u}(s, \eta)) d s d \eta \\
& +\frac{1}{2} \int_{0}^{1}[M(x-s, t)-M(x+s, t)] \vec{\psi}(s) d s \\
& -\int_{0}^{t} M_{x}(x, t-s) D \vec{\varphi}(s) d s+\int_{0}^{t} M_{x}(x-1, t-s) \cdot D \vec{g}(s) d s,
\end{aligned}
$$

where

$$
\vec{g}(t)=\vec{u}(1, t)
$$


Proof: Any solution of Problem I can be represented in the form (cf. [7])

$$
\vec{u}(x, t)=\sum_{i=1}^{4} \vec{u}_{i}(x, t), \quad(x, t) \in \Omega
$$

where

$$
\begin{aligned}
\vec{u}_{1}(x, t) & =\frac{1}{2} \int_{0}^{t} \int_{0}^{1}[M(x-s, t-\eta)-M(x+s, t-\eta)] \vec{f}(s, \eta, \vec{u}(s, \eta)) d s d \eta \\
\vec{u}_{2}(x, t) & =-\int_{0}^{t} M_{x}(x, t-s) D \vec{\phi}(s), d s ; \\
\vec{u}_{3}(x, t) & =\frac{1}{2} \int_{0}^{1}[M(x-s, t)-M(x+s, t)] \dot{\psi}(s) d s \\
\vec{u}_{1}(x, t) & =\int_{0}^{t} M_{x}(x-1, t-s) \vec{u}(1, s) d s .
\end{aligned}
$$

From the nonlocal condition (1.4) it follows that the function $\vec{g}(t)$ given by formula (1.7) satisfies the system of Volterra integral equations of the second kind

$$
i^{\prime} \vec{g}(i)=\vec{F}\left(x_{0}, t\right)+D \int_{0}^{t} M_{x}\left(x_{0}-1, t-s\right)^{\prime} \vec{g}(s) d s,
$$

where

$$
\begin{aligned}
\vec{F}\left(x_{0}, t\right)= & \vec{h}\left(x_{0}, t, \vec{u}\left(x_{0}, t\right)\right)-\int_{0}^{t} M_{x}\left(x_{0}, t-s\right) D \vec{\varphi}(s) d s \\
& +\frac{1}{2} \int_{0}^{1}\left[M\left(x_{0}-s, t\right)-M\left(x_{0}+s, t\right)\right] \vec{\psi}(s) d s \\
& +\frac{1}{2} \int_{0}^{t} \int_{0}^{1}\left[M\left(x_{0}-s, t-\eta\right)-M\left(x_{0}+s, t-\eta\right)\right] \vec{f}(s, \eta, \vec{u}(s, \eta)) d s d \eta
\end{aligned}
$$

The solution of the system (1.8) can be written in the form

$$
\vec{g}(t)=\vec{F}\left(x_{0}^{\prime}, t\right)+\sum_{k=1}^{\infty} A^{k} \vec{F}\left(x_{0}, t\right)
$$

where the operators $A^{k}$ are defined by the formula

$$
A \vec{v}(t) \equiv D \int_{0}^{t} M_{x}\left(x_{0}-1, t-s\right) \vec{v}(s) d s, \quad A^{k} \vec{v}=A\left(A^{k-1} \vec{v}\right) \quad(k=2,3, \ldots)
$$

Thus we have

$$
\vec{u}_{4}(x, t)=\int_{0}^{t} M_{x}(x-1, t-\because s) D\left[\vec{F}\left(x_{0}, s\right)+\sum_{k=1}^{\infty} A^{k}\left(\vec{F}\left(x_{0}, s\right)\right)\right] d s .
$$


Since $x_{0}$ is an internal point of the interval $(0,1)$, the kernel function $M_{x}\left(x_{0}-1, t-s\right)$ is continuous. Therefore, applying Bielecki's theorem [9: p. 31], we can easily' prove that the series $\sum_{k=1}^{\infty} A^{k}(\cdot)$ is convergent (in the norm topology) and that the resolvent of the operator $A$ is of the form (1.10) what completes the proof

Now we sét.

$$
\begin{aligned}
L \vec{u}(x, t)= & \frac{1}{2} \int_{0}^{t} \cdot \int_{0}^{1}[M(x-s, t-\eta)-M(x+s, t-\eta)] \vec{f}(s, \eta, \vec{u}(s, \eta)) d s d \eta \\
& \quad-\int_{0}^{t} M_{x}(x, t-s) D \vec{p}(s) d s+\frac{1}{2} \int_{0}^{1}[M(x-s, t)-M(x+s, t)] \vec{\phi}(s) d s \\
& +\int_{0}^{t} M_{x}(x-1, t-s) D \vec{g}(s) d s, \\
& \|u\|_{\lambda}=\sup _{x, t \in \Omega}\left\{\|u(x, i)\|_{\mathbf{E}_{n}} \mathrm{e}^{-\lambda t}\right\}, \quad \varrho_{\lambda}\left(\vec{u}^{1}, \vec{u}^{2}\right)=\left\|\vec{u}^{1}-\vec{u}^{2}\right\|_{\lambda}, \lambda>0
\end{aligned}
$$

The matrix norm is defined by $\|D\|=\sup \|D \vec{\imath}\| \mathbf{E}_{n}$.

$$
\sum_{i=1}^{n} v_{i}^{2} \leqq 1
$$

Theorem 2: If the vector functions $\vec{\varphi}, \vec{\psi}, \vec{f}, \vec{h}$ and the matrix $D$ satisfy the assumptions $\mathrm{a}_{1}-\mathrm{a}_{14}$ and $T$ is small enough, then the operator $L: C(\bar{\Omega}) \rightarrow C(\bar{\Omega})$ defined by $(1.11)$ has a unique fixed point.

We have to prove that

$$
-\varrho_{\lambda}\left(L \vec{u}^{1}, L \vec{u}^{2}\right) \leqq C_{\lambda}\left(\vec{u}^{1}, \vec{u}^{2}\right), \text { and } C<1 .
$$

To prove inequality (2.1) we shall use the following lemmas.

Lem ma 1: If the vector function $\vec{f}$ satisfies the assumptions $\mathrm{a}_{7}-\mathrm{a}_{9}$, then

$$
\begin{aligned}
& \| \frac{1}{2} \int_{0}^{t} \int_{0}^{1}[M(x-s, t-\eta)-M(x+\dot{s}, t-\eta)] \vec{f}\left(s, \eta, u^{1}(s, \eta)\right) d s d \eta \\
& -\frac{1}{2} \int_{0}^{t} \int_{0}^{1}[M(x-s, t-\eta)-M(x+s, t-\eta)] \vec{f}\left(s, \eta, \vec{u}^{2}(s, \eta) d s d \eta \| \mathrm{e}^{-2 t}\right.
\end{aligned}
$$

$\leqq C_{1} \varrho_{2}\left(\vec{u}^{1}, \vec{u}^{2}\right)$,

where $C_{1} \doteq C_{1}(K,\|D\|, \lambda), K$ is the Lipschitz constant from the assumption $\mathrm{a}_{8}$. Besides $\lim _{\lambda \rightarrow \infty} C_{1}(K,\|D\|, \lambda)=0$.'

Lemma 2: If the matrix $D$ satisfies the assumption $\mathrm{a}_{1}$, then

$$
\int_{0}^{1}\left\|M_{x}(x, s)\right\| d s \leqq C_{2}
$$


for $T<\frac{(2 n-x)^{2}}{4 \alpha}(n=1,2, \ldots ; x \in\langle 0,1\rangle)$, where $C_{2}$ is constant and $\alpha$ is not greater than the dimensional of the matrix $D$.

Lem ma $3:$ If the vector functions $\vec{h}, \vec{f}, \vec{\varphi}, \vec{\psi}$ satisfy the assumptions $\mathrm{a}_{2}-\mathrm{a}_{14}$, then the vector function $\vec{g}$ defined by (1.8) satisfies the inequality

$$
\varrho_{\lambda}\left(\vec{g}^{1}, \vec{g}^{2}\right) \leqq C_{3} \varrho_{2}\left(\vec{u}^{1}, \vec{u}^{2}\right)^{-}
$$

where $\vec{g}^{i}=\ddot{g}\left(x, t, \vec{u}^{i}\right), i=1, \dot{2}$. The constunt $C_{3}$ depends on $C_{1}$ and $T^{\prime}(H-$ the Lipschitz constant from assumption $\mathrm{a}_{13}$ ) and can be written in the form

$$
C_{3}=\left(1+c T \sqrt{T} \mathrm{e}^{c T}\right)\left(H+C_{1}\right),
$$

where $c$ is a positive conistant depending oin the norm $\|D\|$.

I. em ma 4 : If the vector functions $\vec{h}, \vec{f}, \vec{\psi}, \vec{\psi}$ and the matrix $D$ satisfy the assumptions $\mathrm{a}_{1}-\mathrm{a}_{14}$, then

$$
\sup _{t}\left\{\left\|\int_{0}^{t} M_{x}(x-1, t-s) D\left(\vec{g}^{1}(s)-\vec{g}^{2}(s)\right) d s\right\| \mathrm{e}^{-\lambda t}\right\} \leqq C_{4} \varrho_{\lambda}\left(\vec{u}^{1}, \vec{u}^{2}\right),
$$

. where $C_{4}=\|D\| \cdot C_{2} \cdot C_{3}$.

The proofs of the Lemimas $1-4$ result from simple calculations. The Lemmas $1-4$ imply a following one.

Lemma'5: If the assumptions $\mathrm{a}_{1}-\mathrm{a}_{14}$ are satisfied and

then

$$
H<\frac{1}{\|D\| C_{2}\left(1+c T \sqrt{T} \mathrm{e}^{c T}\right)}
$$

$$
\varrho_{\lambda}\left(L \vec{u}^{1}, L \vec{u}^{2}\right) \leqq C \varrho_{\lambda}\left(\vec{u}^{1}, \vec{u}^{2}\right),
$$

where $C=C_{1}+C_{4}$ and for $\hat{\lambda}$ sufficiently large $C<1$.

From Lemna 5 there follows inequality (2.1) and Theorem 2.

Theorem 3: The unique fixed point of the operator $L$ is the required solution of Problem I.

Proof: From the following equalities (cf. [7]) $\lim _{x \rightarrow 0^{+}} \vec{u}_{2}(x, t)=\vec{\phi}(t), \lim _{x \rightarrow 1^{-}} \vec{u}_{2}(x, t)=0$, $\lim _{x \rightarrow 0^{+}} \vec{u}_{3}(x, t)=\lim _{x \rightarrow 1^{-}} \vec{u}_{3}(x, t)=0, \quad \lim _{t \rightarrow 0^{+}} \vec{u}_{3}(x, t)=\vec{\psi}(x), \quad \lim _{x \rightarrow 1^{+}} \vec{u}_{1}(x, t)=\lim _{x \rightarrow 1^{-}} \vec{u}_{1}(x, t)$ $=\lim \vec{u}_{1}(x, t)=0$ and from the properties of the matrix function $M$ it follows that $\vec{u}(x, t)$ is a regular solution of Problem $\mathrm{I}$

\section{Formulation of Problem I}

We shall consider the following Problem II: to find solutions of the system

$$
\vec{v}_{t}=D \vec{v}_{x x}+\vec{F}(x, t, \vec{v}(x ; t)), \quad '(x, t) \in \Omega,
$$

subject to the conditions

$$
\vec{v}(0, t)=\vec{f}_{1}(t), . \quad t \in\langle 0, T),
$$




$$
\begin{aligned}
& \ddot{v}(x, 0)=\vec{f}_{2}(x), \quad x \in\langle 0, \hat{\mathbf{p}}\rangle, \\
& \int_{0}^{1} \vec{v}(x, t) d x=\vec{f}_{3}(t), \quad t \in\langle 0, T\rangle,
\end{aligned}
$$

and.

$$
\vec{f}_{2}(0)=\vec{f}_{1}(0), \quad \vec{f}_{3}(0)=\int_{0}^{1} \vec{f}_{2}(x) d x .
$$

The nonlocal boundary condition is given in the integral forn (3.4). According to the physical situation mentioned above the condition (3.4) is concerned with the increase of the gradients of concentrations of fluids at the end points of the interval $(0,1)$.

Indeed, taking into account a diffusion equation

$$
\vec{v}_{t}=D \vec{v}_{x x}+\vec{F}(x, t, \vec{v}(x, t))
$$

and integrating with respect to $t$ we get

$$
\begin{aligned}
\ddot{v}(x, t) & =\vec{v}(x, 0)+\int_{0}^{t} \vec{v}_{t}(x, \tau) d \tau \\
& =\vec{f}_{2}(x)+\int_{0}^{t} D \vec{v}_{x x}(x, \tau) d \tau+\int_{0}^{t} \vec{F}(x, \tau, \vec{v}(x, \tau)) d \tau .
\end{aligned}
$$

Hence

$$
\begin{aligned}
\int_{0}^{1} \vec{v}(x, t) d x= & \int_{0}^{1} \ddot{f}_{2}(x) d x+D \int_{0}^{t}\left[\vec{v}_{x}(1, \tau)-\vec{v}_{x}(0, \tau)\right] d \tau \\
& +\int_{0}^{1} \int_{0}^{t} \vec{F}\left(x, \tau, \ddot{v}(x, \tau) d \tau d \dot{x}=\dot{f}_{3}(t)\right.
\end{aligned}
$$

which is equivalent to the following differentiated condition because of compatibility condition (3.4):

$$
D\left[\ddot{v}_{x}(1, t)-\vec{v}_{x}(0, t)\right]+\int_{0}^{1} \vec{F}(x, t, \ddot{v}(x, t)) d x=f_{3}{ }^{\prime}(t)
$$

or

$$
\vec{v}_{x}(1, t)-\vec{v}_{x}(0, t)=D^{-1} f_{3}^{\prime}(t)-D^{-1} \int_{0}^{1} \vec{k}(x, t, \ddot{v}(x, t)) d x .
$$

We are looking for solutions of Problem II in the form.

$$
\vec{v}(x, t)=\vec{u}(x, t)+\vec{V}(x, t),
$$

where $\vec{V}(x, t)=(1-2 x) \vec{f}_{1}(t)+2 x \vec{f}_{3}(t)$. This permits to transform Problem $\ddot{\prime}^{\prime}$ II into the form

$$
\begin{array}{ll}
\vec{u}_{t}=D \vec{u}_{x x}+\vec{f}(x, t, \vec{u}), & (x, t) \in \Omega, \\
\vec{u}(0, t)=0, & t \in\langle 0, T), \\
\vec{u}(x, 0)=\vec{\varphi}(x), & x \in\langle 0,1\rangle, \\
\vec{u}_{x}(1, t)-\vec{u}_{x}(0, t)=\vec{G}(t), . & t \in\langle 0, T),
\end{array}
$$


where

$$
\begin{aligned}
& \vec{f}(x, t, \vec{u}) \doteq \vec{F}(x, t, \vec{u}+\vec{V})-(1-2 x) \vec{f}_{1}^{\prime}(t)-2 x f_{3}^{\prime}(t) \\
& \vec{\phi}(x)=\vec{f}_{2}(x)-(1-2 x) \vec{f}_{1}(0)-2 x \vec{f}_{3}(0) \\
& \vec{G}(t)=D^{-1} \vec{f}_{3}^{\prime}(t)-D^{-1} \int_{0}^{1} \vec{F}(x, t, \vec{u}(x, t)+\vec{V}(x, t)) d x \\
& \vec{\phi}(0)=0
\end{aligned}
$$

The problem (3.5)-(3.8) is the nonlocal problem for the 'derivative $u_{x}$ and can be solved similar methods like Problem I.

\section{REFERENCES}

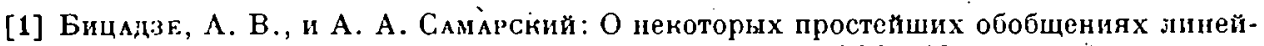
ных әлиптических красвых задач. Докл. Акад. Наук СССР 1S5, 4 (1969), 739-741.

[2] Бжиханов, Х. Г.: Об одной нраевой задаче для смешанных параболо-гиперболических уравнений с характеристической линией, изменения тина. Дифф. Уравн. $13,1(1977), 10-16$.

[3] Эйдельман, С. А.: ПІараболические системы. Москва: Изд-во Наука 1964.

[4] Эидғльман, С. А., и С. А. ИвАсишен: Матрица Грина однородных параболи'ческих граничных задач для системы с разрывными коэффициентами. Докл. Акад. Наук СССР 183, 4 (1968), 797-800.

[5] Ионкин, Н. И., И Е: И. Москев: О задаче для уравнения теплопроводности С двуточечными условиями. Дифф. Уравн. 13, 7 (1979), 1284-1295.

- [6] Керефов, А. А.: Нелокальные краевые задачи для параболических уравнений. Дифф. Уравн. 15, 1 (1979), 74-78.

[7] M $\triangle$ JCHrowSKr, M.: On construction of the parabolic system of partial differential equations. Demonstr. Math. 13 (1980), $285-299$.

[8] Н Апсо, А. Ф.: Об одной нелокальной задаче для уравнения смешаниого парабологиперболического типа. - Дифф. Уравн. 14, 1 (1978), 185-186.

[9] Przeworska-Rolewicz, D.: Equations with transformed argument. An algebraic approach. Elsevier (Amsterdam) and PWN (Warszawa) 1973.

[10] Ройтвегг, Я. А., и Э. Г. МЕфтель: Об одном классе общих нелююалыных элипти.ческих задач. Докл. Акад. 'Наук СССР 192, 3 (1970), $511-514$.

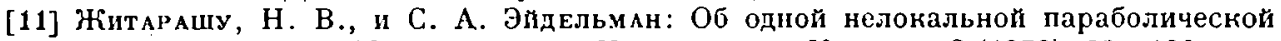
граничной задаче. Математические Исследювания Кишинев 3 (1970), 83-100.

Manuskripteingang: 07. 12. 1982, in revidierter Fassung: 22.04. 1983

\section{VERFÁSSER :}

\section{Dr. Eugeniusz Maciej Chrzanowski}

Institute of Mathematics, Warsaw Technical University

P-00-661 Warszawa, Plac Jedności Robotniczej 1 